Published in Neurophysiologie Clinique, 2019, vol. 49 no. 6, p.

\title{
Caractérisation de la mobilité des visiteurs lors d'une visite muséale aux musée des Beaux-Arts de Montréal
}

Anne-Violette Bruyneel (a), Rachid Aissaoui (b), Serge Mesure (c), Thomas Robert (d), Sylvie Nadeau (e)

(a) Department of Physiotherapy, University of Applied Sciences of Western Switzerland, Genève, Suisse

(b) Laboratoire de recherche en imagerie et orthopédie (LIO), École de Technologie Supérieure, Centre de Recherche du Centre Hospitalier de l'Université de Montréal, H2X 0A9, Montréal, Canada

(c) Aix Marseille Université, Marseille, France

(d) LBMC, Institut français des sciences et technologies des transports de l'aménagement et des réseaux, France

(e) École de réadaptation, Université de Montréal et Centre de Recherche

Interdisciplinaire en Réadaptation (CRIR), Institut Universitaire sur la Réadaptation en

Déficience Physique de Montréal, H3S 1M9, Montréal, Canada

Auteur correspondant : anne-violette.bruyneel@hesge.ch

\section{Introduction :}

L'observation d'œuvres d'art induit un bien-être, une diminution du stress et un effet sur la posture. La visite d'un musée a la particularité de proposer au fil des déplacements moteurs une interaction entre le visiteur et les œuvres dans un environnement pouvant être contraint (ex : obscurité, nombre de personnes, escaliers, ...). La visite muséale constitue une approche culturelle originale pour solliciter les capacités motrices et physiques. L'objectif est de quantifier les exigences neuro-physiologiques lors d'une visite muséale (MBAM). Notre hypothèse est que ces exigences sollicitent suffisamment le corps pour induire des effets bénéfiques sur le système locomoteur et les startégies d'organisation de la motricité.

\section{Matériel et méthodes :}

Trente-cinq sujets seront inclus dans cette étude observationnelle. Les paramètres spatiotemporels de la marche avant et après la visite (Gaitrite), les caractéristiques des mouvements du passage assis-debout, des escaliers et des déplacements (capteurs inertiels) ainsi l'équilibre lors de l'observation d'œuvres 2D et 3D (application téléphonique) seront enregistrés lors de la visite. Les comparaisons avant / après et entre les conditions 
d'équilibre et de stabilisation seront testées. La sollicitation inhérente à la visite sera comparée aux résultats issus de la littérature.

\section{Discussion:}

Cette première étape de caractérisation devrait servir de base pour la compréhension de la sollicitation physique générée lors d'une visite d'une exposition. Par la suite, les résultats devront permettre d'expliquer les éléments ressentis par le visiteur (ex : fatigue), donner une perception différente et bénéfique des visites muséales sur la santé et utiliser ces connaissances sur la mise en place et l'organisation des musées afin de les rendre plus pertinents pour ces facteurs santé.

Mots-clés : Musée - visite - mobilité - équilibre

Les auteurs ne déclarent aucun conflit d'intérêt. 